\title{
A BOREL SOLUTION TO THE HORN-TARSKI PROBLEM
}

\author{
STEVO TODORCEVIC
}

\begin{abstract}
We describe a Borel poset satisfying the $\sigma$-finite chain condition but failing to satisfy the $\sigma$-bounded chain condition.
\end{abstract}

MSC 2000: 03E05, 03E20, 06A10

Keywords: Chain Conditions, Boolean Algebras.

\section{INTRODUCTION}

If a Boolean algebra $\mathbb{B}$ supports a strictly positive finitely additive measure then $\mathbb{B}$ must satisfy the following two standard chain conditions.

The $\sigma$-finite chain condition stating that the collection $\mathbb{B}^{+}$of nonzero elements of $\mathbb{B}$ admits a countable decomposition $\mathbb{B}^{+}=\bigcup_{n<\omega} \mathbb{B}_{n}$ such that no $\mathbb{B}_{n}$ contains infinitely many pairwise disjoint elements.

The $\sigma$-bounded chain condition stating that $\mathbb{B}^{+}$admits a countable decomposition $\mathbb{B}^{+}=\bigcup_{n<\omega} \mathbb{B}_{n}$ such that for every $n<\omega$ there is an integer $k_{n}$ such that $\mathbb{B}_{n}$ contains no more than $k_{n}$ pairwise disjoint elements.

These two chain conditions were first considered by Horn and Tarski who asked ([3], p.482) whether they are actually equivalent in the class of all Boolean algebras. This problem is closely related to the more well-known problem asking whether a Boolean algebra can support an exhaustive but not uniformly exhaustive strictly positive submeasure (see [5]). To see this note that the $\sigma$-finite chain condition on $\mathbb{B}$ is equivalent to the existence of a functional $\varphi: \mathbb{B} \rightarrow[0, \infty)$ which is strictly positive, i.e., $\varphi(a)=0$ iff $a=0$ and which is exhaustive in the sense that $\lim \varphi\left(a_{n}\right)=0$ for every infinite sequence $\left(a_{n}\right)$ of pairwise disjoint elements of $\mathbb{B}$. On the other hand, the $\sigma$-bounded chain condition on $\mathbb{B}$ is equivalent to the existence of a strictly positive functional $\varphi: \mathbb{B} \rightarrow[0, \infty)$ that is uniformly exhaustive in the sense that for every $\varepsilon>0$ there is an integer $k_{\varepsilon}$ such that for every sequence $\left\langle a_{n}: n<k_{\varepsilon}\right\rangle$ of pairwise disjoint elements of $\mathbb{B}$ there must be an $n<k_{\varepsilon}$ such that $\varphi\left(a_{n}\right)<\varepsilon$. Recall that that Maharam's problem was solved by Talagrand [7] who constructed an exhaustive but not uniformly exhaustive 
strictly positive submeasure on the countable atomless Boolean algebra. Unfortunately, Talagrand's construction did not solve the Horn-Tarski problem. This has been done in a recent paper by Thümmel [8] who besides a number of new ideas used also some ideas from our paper [9] in order to construct an algebra that distinguishes between the two conditions of Horn and Tarski. As the title of [9] suggests, our paper was devoted to the distinctions between various chain condition of posets from the descriptive set-theoretic perspective, a perspective that could be of independent interest (see, for example, [2]). The purpose of this note is to complete this line of investigation by proving the following result.

1.1 Theorem. There is a Borel partially ordered set $\mathcal{T}(\pi \mathbb{Q})$ that satisfies the $\sigma$-finite chain condition but fails to satisfy the $\sigma$-bounded chain condition.

As the notation suggest we use here another idea from [9], a pseudotree structure $\pi(\mathbb{Q})$ on the family of all bounded sets of rationales. In showing that $\mathcal{T}(\pi \mathbb{Q})$ does not satisfy the $\sigma$-bounded chain condition we do rely on the key idea of Thümmel [8] but the subtle difference is that the arguments of [8] depend on the fact that Thümmel's tree (actually a linear ordering) is $(\omega, \infty)$-distributive while our pseudo-tree $\pi \mathbb{Q}$ is not. In order to deal with this we had to introduce another idea, a suitable Borel topology on $\pi(\mathbb{Q})$.

We should also point out that there are concrete internal reasons for dealing with the Borel version of the Horn-Tarski problem. In fact, they comes from various attempts to provide a positive answer to the Horn-Tarski problem by induction on the natural rank coming from the $\sigma$-finite chain condition which in the Borel case could perhaps be subject to various boundedness theorems of descriptive set theory. The reader interested in this will notice that our decomposition of the Borel poset $\mathcal{T}(\pi \mathbb{Q})$ witnessing the $\sigma$-finite chain condition is not Borel itself, and in fact cannot be Borel. Yet another motivation for examining the Borel version of the Horn-Tarski problem could be found by recalling what happened in the case of the closely related problem of Maharam [6] about the existence of a continuous strictly positive submeasure on a given $\sigma$-complete Boolean algebra $\mathbb{B}$. Namely, while Souslin algebra serves as a counterexample to Maharam's list of conditions ${ }^{1}$ to guarantee the existence of such a submeasure, in the Borel case her problem has always a positive answer (see [10]). Finally, we mention that the following problem suggests itself.

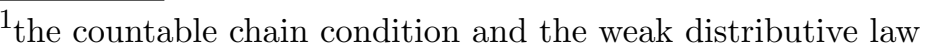


1.2 Problem. Is there a complete Boolean algebra $\mathbb{B}$ supporting a continuous strictly positive submeasure but failing to satisfy the $\sigma$-finite chain condition?

We finish the introduction by reviewing some standard notation and terminology. Recall, that every partially ordered set $\mathcal{P}$ determines the complete Boolean algebra $\mathbb{B}=\mathbb{B}(\mathcal{P})$ consisting of regular-open subsets of $\mathcal{P}$ in the topology generated by $(-\infty, p](p \in \mathcal{P})$ (see for, example, [4]). When $\mathcal{P}$ is separative, i.e., when $p \not \leq q$ implies that there is $r \leq p$ such that $r \perp q^{2}$, then $\mathcal{P}$ is isomorphic to a dense subset of $\mathbb{B}^{+}$. Then incompatibility relation $x \perp y$ of $\mathcal{P}$ transfers to the disjointedness relation $x \wedge y=0$ on $\mathbb{B}=\mathbb{B}(\mathcal{P})$ and essentially all of the chain conditions on $\mathbb{B}=\mathbb{B}(\mathcal{P})$ transfer to chain conditions on $\mathcal{P}$ and vice versa. In Forcing one usually find it more convenient to work with posets rather than the corresponding Boolean algebras. For example, the notion of a Borel poset $\mathcal{P}=(P, \leq)$ is much more natural in this context as it simply mean that the domain $P$ is a Borel subset of some Polish space, that the order relation $\leq$ is a Borel subset of $P^{2}$ and that, moreover, the incompatibility relation $\perp$ is also a Borel subset of $P^{2}$ (see [2]). The rest of our terminology and notation is quite standard and can be found in texts like [2] and [4].

\section{A topology on $\pi \mathbb{Q}$}

Let $\pi \mathbb{Q}$ be the collection of all bounded subsets of the set $\mathbb{Q}$ or rational numbers that are either empty or have minimal elements. We order $\pi \mathbb{Q}$ by letting $s \leq t$ if either $s=t$ or $s$ is an initial segment of $t$ (in notation $s \sqsubset t$ ) and such that $\min (t \backslash s)$ exists. Note that $\pi \mathbb{Q}$ is a partially ordered set whose minimal element is the empty set and which has the property that for every $t \in \pi \mathbb{Q}$, the set

$$
\operatorname{Pred}(t)=\{s \in \pi \mathbb{Q}: s<t\}
$$

of predecessors of $t$ is a countable totally ordered set and the set

$$
\operatorname{ImmSucc}(t)=\{t \cup\{q\}: q \in \mathbb{Q}, q>\sup (t)\}
$$

of immediate successors of $t$ is also a countable set. Note that $\pi \mathbb{Q}$ is an $\sigma$-compact subset of $2^{\mathbb{Q}}$ and that $\leq$ is a $F_{\sigma}$ relation on $\pi \mathbb{Q}$. Given $t \in \pi \mathbb{Q}$ and $q>\sup (t)$ let

$$
B_{t}(q)=\{s \in \pi \mathbb{Q}: s \sqsupseteq t \text { and } \sup (s)<q\} .
$$

\footnotetext{
${ }^{2}$ Here, $\perp$ denotes the incompatibility relation on $\mathcal{P}$, i.e. $x \perp y$ iff there is no $z$ such that $z \leq x$ and $z \leq y$.
} 
Note that $B_{t}(q)(t \in \pi \mathbb{Q}, q>\sup (t))$ forms a basis for a first countable topology on $\pi \mathbb{Q}$. From now on, unless otherwise stated, it is this topology on $\pi \mathbb{Q}$ that we would be referring to. For example, the following set defined using this topology plays an important role in the rest of the paper,

$$
\mathcal{S}_{\omega}(\pi \mathbb{Q})=\left\{S \subseteq \pi \mathbb{Q}:|S|=\aleph_{0} \text { and }\left|S^{(1)}\right|=1\right\},
$$

where for $S \subseteq \pi \mathbb{Q}$, the $S^{(1)}$ denotes the collection of all $x \in S$ that are not isolated in $S$ with the subspace topology induced from $\pi \mathbb{Q}$. Thus $\mathcal{S}_{\omega}(\pi \mathbb{Q})$ is simply the collection of infinite countable converging sequences $S$ in $\pi \mathbb{Q}$ including their limit points $\lim (S)$. We shall use the notation $S \rightarrow \lim (S)$ to indicate this. Note that $\mathcal{S}_{\omega}(\pi \mathbb{Q})$ can be coded as a Borel subset of the Polish space $\left(2^{\mathbb{Q}}\right)^{\mathbb{N}}$.

\section{A Borel poset}

Let $\mathcal{T}(\pi \mathbb{Q})$ be the collection of all countable compact subsets $K$ of $\pi \mathbb{Q}$ with $K^{(1)}$ finite. We order Let $\mathcal{T}(\pi \mathbb{Q})$ by letting $K \leq L$ if $K \supseteq L$ and

$$
K^{(1)} \cap L=L^{(1)} .
$$

Note that $\mathcal{T}(\pi \mathbb{Q})$ can be coded as a Borel subset of the Polish space $\left(2^{\mathbb{Q}}\right)^{\mathbb{N} \times \mathbb{N}}$ with both order and the incompatibility relations interpreted as Borel subsets of its square. Note also that $\mathcal{T}(\pi \mathbb{Q})$ has a separative version (see [1]) which is also Borel. More precisely, the separative version of $\mathcal{T}(\pi \mathbb{Q})$ is the collection of all functions $P: D_{P} \rightarrow 2$ such that $D_{P}$ is a countable compact subset of $\pi \mathbb{Q}$ with $D_{P}^{(1)}$ finite such that

$$
D_{P}^{(1)} \subseteq P^{-1}(1) \text { and }\left|P^{-1}(1)\right|<\aleph_{0}
$$

where the ordering is given by $P \leq Q$ iff $D_{P} \supseteq D_{Q}$ and $Q=P \uparrow D_{Q}$. It should be clear that for our purposes here it does not matter which of the two versions of $\mathcal{T}(\pi \mathbb{Q})$ we analyze. We choose the first one simply because of the notational convenience.

\section{The $\sigma$-BOUNDED CHAIN CONDITION}

The purpose of this section is to prove the following fact.

4.1 Lemma. The poset $\mathcal{T}(\pi \mathbb{Q})$ does not satisfy the $\sigma$-bounded chain condition.

Proof. Suppose that there is a decomposition $\mathcal{T}(\pi \mathbb{Q})=\bigcup_{n<\omega} \mathcal{T}_{n}$ such that for every $n<\omega$, the family $\mathcal{T}_{n}$ contains no subfamily of size $n+2$ 
consisting of pairwise incompatible elements of $\mathcal{T}(\pi \mathbb{Q})$. For $t \in \pi \mathbb{Q}$ and $n<\omega$, and $q>\sup (t)$ let

$$
\mathcal{T}_{n}(t, q)=\left\{K \in \mathcal{T}:\left(\exists s \in K^{(1)}\right) s \sqsupseteq t \text { and } \sup (s)<q\right\},
$$

and let $\varphi_{n}(t, q)$ be the largest integer $k<n+2$ for which we can find an incompatible subfamily of $\mathcal{T}_{n}(t, q)$ of cardinality $k$. Note that $\varphi_{n}(t, q) \geq \varphi_{n}(u, r)$ whenever $t \sqsubseteq u$ and $q \geq r$.

4.1.1 Claim. For all $s \in \pi \mathbb{Q}, n<\omega$ and $p>\sup (s)$ there is $t \sqsupseteq s$ and $q \in(\sup (t), p)$ such that $\varphi_{n}(u, r)=\varphi_{n}(t, q)$ for all $u \sqsupseteq t$ and $r \leq q$ such that $\sup (u)<r$.

Proof. Otherwise, we get an infinite decreasing sequence of positive integers $<n+2$.

Using the Claim we can build an $\sqsubset$-increasing sequence $t_{n}(n<\omega)$ of elements of $\pi \mathbb{Q}$ and a decreasing sequence $q_{n}(n<\omega)$ of rationals such that for all $n<\omega$

(1) $\sup \left(t_{n}\right)<q_{n}$,

(2) $\varphi_{n}\left(u, q_{n}\right)=\varphi_{n}\left(t_{n}, q_{n}\right)$ for all $u \sqsupseteq t_{n}$ such that $\sup (u)<q_{n}$.

Moreover, we arrange that

$$
\sup _{n<\omega} \sup \left(t_{n}\right)=\inf _{n<\omega} q_{n} .
$$

Let $t_{\omega}=\bigcup_{n<\omega} t_{n}$. By (2), for each $n<\omega$, we can choose $\mathcal{X}_{n} \subseteq$ $\mathcal{T}_{n}\left(t_{\omega} \cup\left\{q_{n+1}\right\}, q_{n}\right)$ of cardinality $\varphi_{n}\left(t_{n}\right)$ consisting of pairwise incompatible conditions of $\mathcal{T}(\pi \mathbb{Q})$. Then for each $K \in \mathcal{X}_{n}$ we can pick $x_{n}^{K} \in K^{(1)}$ such that $x_{n}^{K} \sqsupseteq t_{\omega} \cup\left\{q_{n+1}\right\}$ and $\sup \left(x_{n}^{K}\right)<q_{n}$.

Let

$$
L=\left\{t_{\omega}\right\} \cup\left\{x_{n}^{K}: n<\omega, K \in \mathcal{X}_{n}\right\} \cup\left\{t_{\omega} \cup\left\{q_{n}\right\}: n<\omega\right\} .
$$

Then $L$ is an element of our poset $\mathcal{T}(\pi \mathbb{Q})$ with unique non-isolated point $t_{\omega}$, i.e., a converging sequence with its limit point, a member of $\mathcal{S}_{\omega}(\pi \mathbb{Q})$. Fix $m<\omega$ such that $L \in \mathcal{T}_{m}$. Note that since $L$ belongs to $\mathcal{T}_{m}\left(t_{m}, q_{m}\right)$, we have that $\varphi_{m}\left(t_{m}, q_{m}\right)>0$ and so $\mathcal{X}_{m} \neq \emptyset$. On the other hand, note that by the choice of $L$ we have that

$$
\left\{x_{m}^{K}: K \in \mathcal{X}_{m}\right\} \subseteq L \backslash L^{(1)} .
$$

This means that $L$ is incompatible with every element of $\mathcal{X}_{m}$. Since $L$ belongs to $\mathcal{T}_{n}\left(t_{m}, q_{m}\right)$ this contradicting the maximality of the integer $\varphi_{m}\left(t_{m}, q_{m}\right)=\left|\mathcal{X}_{m}\right|$. This finishes the proof. 


\section{The $\sigma$-FINITE CHAIN CONDITION}

In this section we prove the following fact.

5.1 Lemma. The poset $\mathcal{T}(\pi \mathbb{Q})$ satisfies the $\sigma$-finite chain condition.

Proof. Fix a well-ordering $<_{w}$ of $\pi \mathbb{Q}$ and an enumeration $\left\{q_{k} ; k<\omega\right\}$ of $\mathbb{Q}$. Then for each $t \in \pi \mathbb{Q}$, we can let $v(t)$ denote the $<_{w}$-minimal $v \in \pi \mathbb{Q}$ such that $v \sqsupset t$. For $k<\omega$, let

$$
\pi_{k} \mathbb{Q}=\left\{t \in \pi \mathbb{Q}: q_{k}=\min (v(t) \backslash t)\right\} .
$$

Then $\pi \mathbb{Q}=\bigcup_{k<\omega} \pi_{k} \mathbb{Q}$. Note that for each $k<\omega$, the relation $\sqsubseteq$ of end-extension is well-founded on $\pi_{k} \mathbb{Q}$.

Given $K \in \mathcal{T}(\pi \mathbb{Q})$, since $K^{(1)}$ is a finite subset of $\pi \mathbb{Q}$, we can let $b(K)$ be the minimal integer $b$ such that $K^{(1)} \subseteq \bigcup_{k<b} \pi_{k} \mathbb{Q}$. Let $l(K)=$ $\left|K^{(1)}\right|$. The enumeration $q_{k}$ of $\mathbb{Q}$ in order type $\omega$ gives us a natural lexicographical ordering of $\pi \mathbb{Q}$, so let $x_{i}^{K}(i<l(K))$ be the listing of $K^{(1)}$ according to this ordering. Then we can pick a sequence $\left\langle q_{i}^{K}\right.$ : $i<l(K)\rangle \subseteq \mathbb{Q}$ of such that $q_{i}^{K}>\sup \left(x_{i}^{K}\right)$ for all $i<l(K)$ and such that $B_{x_{i}^{K}}\left(q_{i}^{K}\right)(i<l(K))$ is a sequence of pairwise disjoint basic open neighborhoods. Moreover, we arrange that for $i, j<l(K)$,

(1) $\sup \left(x_{i}^{K}\right)<\sup \left(x_{j}^{K}\right)$ implies $q_{i}^{K}<\sup \left(x_{j}^{K}\right)$ and

(2) $\sup \left(x_{i}^{K}\right)=\sup \left(x_{j}^{K}\right)$ implies $q_{i}^{K}=q_{j}^{K}$.

Note that the set $D(K)=K \backslash \bigcup_{i<l(K)} B_{x_{i}^{K}}\left(q_{i}\right)$ must be finite, so we let $d(K)$ be its cardinality.

For $b, d, l<\omega$ and $\left\langle q_{i}: i<l\right\rangle \subseteq \mathbb{Q}$, let $\mathcal{X}\left(b, d, l,\left\langle q_{i}: i<l\right\rangle\right)$ be the set

$$
\left\{K \in \mathcal{T}(\pi \mathbb{Q}): b(K)=b, d(K)=d, l(K)=l, q_{i}^{K}=q_{i} \text { for all } i<l\right\} .
$$

The following Claim finishes the proof.

5.1.1 Claim. For all $b, d, l<\omega$ and $\left\langle q_{i}: i<l\right\rangle \subseteq \mathbb{Q}$, the set

$$
\mathcal{X}\left(b, d, l,\left\langle\left(p_{i}, q_{i}\right): i<l\right\rangle\right)
$$

contains no infinite sequence of pairwise incompatible elements.

Proof. Let $K_{n}(n<\omega)$ be a given infinite sequence of elements of $\mathcal{X}\left(b, d, l,\left\langle q_{i}: i<l\right\rangle\right)$. We need to find $m<n$ with $K_{m}$ and $K_{n}$ compatible in $\mathcal{T}(\pi \mathbb{Q})$. Since the sets $D\left(K_{n}\right) \cup K_{n}^{(1)}$ are of uniformly bounded cardinality $d+l$, by a simple application of Ramsey theorem and going to an infinite subsequence, we may assume that $D\left(K_{n}\right) \cup K_{n}^{(1)}(n<\omega)$ forma a $\Delta$-system with root $D \cup E$. This in particular means that $D\left(K_{m}\right) \cap K_{n}^{(1)}=\emptyset$ for $m \neq n$ and, therefore, that we can concentrate 
on the case $d=0$, i.e., the case when $D\left(K_{n}\right)=\emptyset$ for all $n$. For $n<\omega$ and $i<l$, let

$$
K_{n}(i)=K_{n} \cap B_{x_{i}^{K_{n}}}\left(q_{i}\right) .
$$

Then $K_{n}(i)(i<l)$ is a sequence of pairwise disjoint converging sequences that cover the set $K_{n}$. Since $\left\{x_{i}^{K_{n}}: i<l, n<\omega\right\}$ is a subset of the well-founded poset $\left(\bigcup_{k<b} \pi_{k} \mathbb{Q}, \sqsubseteq\right)$, refining further, we may assume that for all $i<l$, the sequence $x_{i}^{K_{n}}(n<\omega)$ is either strictly $\sqsubseteq$-increasing, or it forms an $\sqsubseteq$-antichain.

Suppose, by way to a contradiction, that for all $m \neq n$, the sets $K_{m}$ and $K_{n}$ are incompatible in $\mathcal{T}(\pi \mathbb{Q})$. Then applying Ramsey theorem, we can find an infinite subset $M$ of $\omega$ and integers $i, j<l$ such that either

(3) $x_{i}^{K_{m}} \in K_{n}(j)$ for all $m<n$ in $M$, or

(4) $x_{i}^{K_{n}} \in K_{m}(j)$ for all $m<n$ in $M$.

Not that in the case (3), we have that $x_{j}^{K_{n}} \sqsubset x_{i}^{K_{m}}$, and so in particular the sequence $x_{j}^{K_{n}}(n<\omega)$ is strictly $\sqsubseteq$-increasing. If for $m<\omega$ we take $n(m)=\min \left(M \backslash\{0, \ldots, m\}\right.$, then we get $x_{j}^{K_{m}} \sqsubset x_{j}^{K_{n(m)}} \sqsubset x_{i}^{K_{m}}$, and therefore $x_{j}^{K_{m}} \sqsubset x_{i}^{K_{m}}$. So, in particular, $\sup \left(x_{j}^{K_{m}}\right)<\sup \left(x_{i}^{K_{m}}\right)$, which by (1) means that $\sup \left(x_{j}^{K_{m}}\right)<q_{j}<\sup \left(x_{i}^{K_{m}}\right)<q_{i}$ for all $m \in M$. This, however, gives us a contradiction since the set $K_{n}(j)$, being included in $B_{x_{j}^{K}}\left(q_{j}\right)$, can't contain $x_{i}^{K_{m}}$ for any $m$. This contradiction eliminates the case (3) and so we are left with the case (4). First of all note that in this case $x_{j}^{K_{m}} \sqsubset x_{i}^{K_{n}}$, an so in particular $x_{j}^{K_{m}}(m<\omega)$ is strictly 巨-increasing. Let $m_{0}<m_{1}$ be the first two elements of $M$ and let

$$
q=\min \left(x_{j}^{K_{m_{1}}} \backslash x_{j}^{K_{m_{0}}}\right) .
$$

Then for $n \in M, n>m_{1}$ we have that $x_{i}^{K_{n}} \sqsupset x_{j}^{K_{m_{1}}}$ and therefore $q=\min \left(x_{i}^{K_{n}} \backslash x_{j}^{K_{m_{0}}}\right)$. It follows that, in particular, the sequence $\left(x_{i}^{K_{n}}\right)$ $\left(n \in M, n>m_{1}\right)$ cannot accumulate to $x_{j}^{K_{m_{0}}}$. But this contradicts the fact that this is an infinite subset of a sequence $K_{m_{0}}(j)$ which converges to $x_{j}^{K_{m_{0}}}$. This contradiction finishes the proof.

\section{REFERENCES}

[1] B. Balcar, T. Pazak and E. Thümmel. On Todorcevic orderings. preprint, 2012.

[2] T. Bartoszyński. Set theory. A K Peters Ltd., Wellesley, MA, 1995. On the structure of the real line. 
[3] A. Horn and A. Tarski. Measures in Boolean algebras. Trans. Amer. Math. Soc., 64:467-497, 1948.

[4] T. Jech. Set theory. Springer Monographs in Mathematics. Springer-Verlag, Berlin, 2003. The third edition, revised and expanded.

[5] N. Kalton. The Maharam problem. In Séminaire d'Initiation à l'Analyse, volume 94 of Publ. Math. Univ. Pierre et Marie Curie, pages Exp. No. 18, 13. Univ. Paris VI, Paris, 1989.

[6] Dorothy Maharam. An algebraic characterization of measure algebras. Ann. of Math. (2), 48:154-167, 1947.

[7] M. Talagrand. Maharam's problem. Ann. of Math. (2), 168(3):981-1009, 2008.

[8] E. Thümmel. The problem of Horn and Tarski. preprint 2012.

[9] S. Todorcevic. Two examples of Borel partially ordered sets with the countable chain condition. Proc. Amer. Math. Soc., 112(4):1125-1128, 1991.

[10] Stevo Todorcevic. A problem of von Neumann and Maharam about algebras supporting continuous submeasures. Fund. Math., 183(2):169-183, 2004. 